\title{
Perspectiva do cuidador familiar frente a criança com anemia falciforme: uma revisão integrativa
}

\author{
Family caregiver's perspective on children with sickle cell disease: an integrative review \\ Perspectiva del cuidador familiar sobre niños con anemia de células falciformes: una revisión \\ integradora
}

6

Recebido: 22/08/2021 | Revisado: 28/08/2021 | Aceito: 01/09/2021 | Publicado: 04/09/2021

Allan Bruno Alves de Sousa Santos

ORCID: https://orcid.org/0000-0001-6412-7164

Faculdade de Educação São Francisco, Brasil

E-mail: abass@faesf.com.br

Lina Eduarda Silva Costa

ORCID: https://orcid.org/0000-0002-9992-4881

Faculdade Carajás, Brasil

E-mail: linaeduardacosta@gmail.com

Kaline Oliveira de Sousa

ORCID: https://orcid.org/0000-0002-7193-4033

Universidade Federal de Campina Grande, Brasil

E-mail: kaline.academico@gmail.com

Bruno Abílio da Silva Machado

ORCID: https://orcid.org/0000-0003-1759-0206

Centro Universitário Maurício de Nassau, Brasil

E-mail: bruno.smachado@ufpe.br

Gabriella Pacheco Costa

ORCID: https://orcid.org/0000-0002-4370-031X

Universidade de Vassouras, Brasil

E-mail: gabriellapacheco_@hotmail.com

Dalton Ferreira Matos

ORCID: https://orcid.org/0000-0002-6188-7536

Universidade Federal de Sergipe, Brasil

E-mail: daltonmatosgn@gmail.com

Livia Maria Tavares Miranda

ORCID: https://orcid.org/0000-0002-7638-6208

Faculdade Venda Nova do Imigrante, Brasil

E-mail: livia.miranda12@gmail.com

Tereza Cristina Santos Gomes de Araújo ORCID: https://orcid.org/0000-0003-2635-1370

Faculdade São Francisco de Juazeiro, Brasil

E-mail: cristina.tcsg@gmail.com

Thiemmy de Souza Almeida Guedes

ORCID: https://orcid.org/0000-0003-2261-0320

Faculdade Venda Nova do Imigrante, Brasil

E-mail: thiemmyalmeida@gmail.com

Victória Maria Pontes Martins

ORCID: https://orcid.org/0000-0002-8281-0132

Centro Universitário INTA, Brasi

E-mail: victoriapontes2014@ hotmail.com

Rafaela de Lima da Cruz

ORCID: https://orcid.org/0000-0003-1067-8678

Universidade Federal de Pelotas, Brasil

E-mail: rafaeladelimacruz.rlc@gmail.com

Débora de Oliveira Lima

ORCID: https://orcid.org/0000-0002-8706-9451

Universidade Federal do Piauí, Brasil

E-mail: deboralima209@gmail.com

Luana Pereira Ibiapina Coêlho

ORCID: https://orcid.org/0000-0002-2054-959X

Universidade Estadual do Maranhão, Brasil

E-mail: luana_ibiapina@hotmail.com

Leandro Barbosa Teixeira

ORCID: https://orcid.org/0000-0002-9444-7828

Universidade Estácio de Sá, Brasil

E-mail: Lbtteixeira@hotmail.com 
Edla Nayara da Silva Lima

ORCID: https://orcid.org/0000-0003-0384-2599 Faculdade M-educar FAMED, Brasil

E-mail: edlanayaras1@hotmail.com

Joyce Quirino da Silva

ORCID: https://orcid.org/0000-0002-2815-4445

Universidade Federal de Pernambuco, Brasil

E-mail: joycequirino021@gmail.com

Allan Francisco Costa Jaques

ORCID: https://orcid.org/0000-0002-7097-5976

Universidade Federal de Pernambuco, Brasil

E-mail: allanjaques1@gmail.com

Saulo Leite de Paula

ORCID: https://orcid.org/0000-0002-9511-6115 Universidade Federal do Ceará, Brasil

E-mail: Saulolp@yahoo.com.br

Yanna Cavalcante Martins

ORCID: https://orcid.org/0000-0002-8291-7757 Faculdade Maurício de NASSAU, Brasil E-mail: Cavalcantedepaulay@gamil.com

Jônatas Lucas Marcelino da Silva

ORCID: https://orcid.org/0000-0002-4063-2104

Universidade Federal de Pernambuco, Brasil

E-mail: jonatas.lucas@ufpe.br

Raphaela da Silva Faria

ORCID: https://orcid.org/0000-0002-5549-7152 IMEPAC Centro Universitário, Brasil

E-mail: raphaela.faria @aluno.imepac.edu.br

Kleber Luiz Santana dos Santos

ORCID: https://orcid.org/0000-0003-1781-6391

Universidade Cesumar, Brasil

E-mail: santanared@gmail.com

Luciana Carneiro de Brito Ramalho

ORCID: https://orcid.org/0000-0002-4258-8143

Faculdade Bezerra de Araújo, Brasil E-mail: luciana016@gmail.com

Gerson Pedroso de Oliveira

ORCID: https://orcid.org/0000-0002-3532-887X Centro de Pós Graduação e Aperfeiçoamento LTDA, Brasil E-mail: gerson-06@hotmail.com

\section{Resumo}

Este estudo tem como objetivo, buscar na literatura as atividades realizadas pelos cuidadores para promover a criança com anemia falciforme uma melhor qualidade de vida, enfatizando a importância da assistência domiciliar. Trata-se de um estudo descritivo do tipo revisão integrativa da literatura. A coleta ocorreu nas bases de dados: Literatura Latino-Americana e do Caribe em Ciências da Saúde (LILACS); base de Dados de Enfermagem (BDENF) e portal Scientific Electronic Library Online (SciELO). Os estudos foram coletados nos períodos de junho a julho de 2021, usados os descritores "Cuidadores; criança; Anemia Falciforme", cruzados com os operadores booleanos "AND" e "OR", localizados na lista dos Descritores em Ciências da Saúde (DECs). Após aplicação dos critérios de elegibilidade, foram selecionados 9 estudos para a pesquisa. A principal dificuldade evidenciada nesse estudo, foi a falta de informações corretas dos cuidadores acerca da anemia falciforme, e a falta de interação entre profissionais da saúde e familiares, apontou-se efetividade nas atividades diárias de cuidado, como alimentação e higiene, e o manejo da dor nas crianças. Conclui-se que é necessária uma educação em saúde eficaz para essa população, pois a informação correta sobre a doença, é a base para uma assistência de qualidade no ambiente domiciliar.

Palavras-chave: Cuidadores; Criança; Anemia falciforme.

\section{Abstract}

This study aims to search the literature for activities performed by caregivers to promote a better quality of life for children with sickle cell anemia, emphasizing the importance of home care. This is a descriptive study of the integrative literature review type. The collection took place in the following databases: Latin American and Caribbean Literature on Health Sciences (LILACS); Nursing Database (BDENF) and Scientific Electronic Library Online (SciELO). The studies were collected from June to July 2021, using the descriptors "Caregivers; kid; Sickle Cell Anemia", crossed with the Boolean operators "AND" and "OR", located in the list of Health Science Descriptors (DECs). After applying the eligibility criteria, 9 studies were selected for the research. The main difficulty highlighted in this study was the lack of correct information from caregivers about sickle cell anemia, and the lack of interaction between health professionals and family members. of pain in children. It is concluded that an effective 
health education is needed for this population, as correct information about the disease is the basis for quality care in the home environment.

Keywords: Caregivers; Kid; Sickle cell anemia.

\section{Resumen}

Este estudio tiene como objetivo buscar en la literatura las actividades que realizan los cuidadores para promover una mejor calidad de vida de los niños con anemia falciforme, enfatizando la importancia del cuidado domiciliario. Se trata de un estudio descriptivo del tipo revisión integradora de la literatura. La colección se llevó a cabo en las siguientes bases de datos: Literatura Latinoamericana y del Caribe en Ciencias de la Salud (LILACS); Base de datos de enfermería (BDENF) y Biblioteca electrónica científica en línea (SciELO). Los estudios se recopilaron de junio a julio de 2021, utilizando los descriptores "Cuidadores; niño; Anemia de células falciformes", cruzada con los operadores booleanos "Y" y "O", ubicados en la lista de Descriptores de Ciencias de la Salud (DEC). Después de aplicar los criterios de elegibilidad, se seleccionaron 9 estudios para la investigación. La principal dificultad que se destacó en este estudio fue la falta de información correcta por parte de los cuidadores sobre la anemia de células falciformes y la falta de interacción entre los profesionales de la salud y los familiares del dolor en los niños. Se concluye que es necesaria una educación sanitaria eficaz para esta población, ya que la información correcta sobre la enfermedad es la base de una atención de calidad en el ámbito del hogar.

Palabras clave: Cuidadores; Niño; Anemia de células falciformes.

\section{Introdução}

Segundo o Ministério da saúde, a anemia falciforme (AF), é conceituada como uma patologia genética e hereditária, na qual ocorre uma mutação que ocasiona alterações nos formatos das hemácias, deixando-as em formato que lembra uma "foice", isso dificulta o carreamento de oxigênio aos tecidos o que gera ao organismo uma seria de malefícios, dentre eles, dor crônica, baixa imunidade, icterícia, dentre outros (Ministério da saúde, 2020).

O Sistema Único de Saúde (SUS), disponibiliza para a população o tratamento farmacológico completo, objetivando melhorar a qualidade de vida dessas pessoas, dentre esses medicamentos podemos citar: ácido fólico, anti-inflamatórios, analgésicos, antibióticos e a hidroxiuréia, principal medicamento promotor de qualidade de vida a pessoa com doença falciforme (Ministério da saúde, 2020).

O Ministério da saúde estima que existam no Brasil, um quantitativo de 30 a 50 mil pessoas com alguma doença falciforme, sendo as regiões norte e nordeste as mais afetadas, já sua prevalência a nível global, aponta-se que a cada ano seja diagnosticado entre 300 a 500 mil novos casos, sendo a África o continente de maior prevalência, com o quantitativo estimado de 230 mil casos (Kanto et al., 2018).

Existem estratégias ofertada pelas políticas públicas, do governo brasileiro, voltadas para as populações que possuem doenças falciformes, dentre elas a AF, no entanto a precariedade das informações ofertadas a população, não permite que essas informações cheguem nos locais mais distantes, e ocasionando assim a exclusão da população mais carente as terapias farmacológicas e assistência especializada (Silva et al, 2013).

A teoria do cuidado humano está centrada nos conceitos de cuidados holísticos, na qual vai muito além de apenas a dimensão física, mais também as dimensões culturais, sociais espirituais, objetivando sempre promover ao paciente, as melhores condições para o desenvolvimento de uma boa qualidade de vida (Favero, Meier, Lacerda, Mazza \& Kalinowski, 2009).

Assim, o cuidador familiar desempenha papel crucial na promoção de qualidade de vida ao paciente com AF, por se tratar de uma doença de caráter crônico, requer cuidados integrais e intensivos, e a entrega dos envolvidos na assistência, afim de evitar as complicações da patologia, portanto é indispensável o envolvimento dos cuidadores, considerando que convivem diariamente desde o diagnostico até o passar das fases do desenvolvimento humano (Silva et al, 2013). 
O estudo aponta o seguinte objetivo: Buscar na literatura as atividades realizadas pelos cuidadores para promover a criança com anemia falciforme uma melhor qualidade de vida, enfatizando a importância da assistência domiciliar.

\section{Metodologia}

Trata-se de um estudo de caráter descritivo, do tipo revisão integrativa. O estudo integrativo é um método que tem como principal finalidade sintetizar resultados obtidos através de pesquisas, oferecendo os resultados de maneira sistemática e abrangente (Ercole, Melo, Alcoforado, 2014).

Foram seguidas as seguintes etapas para elaboração do estudo: definição do tema e elaboração da problemática através da estratégia PIco (estabelecendo a população de crianças com anemia falciforme; com o interesse em descrever as atividades dos cuidadores domiciliares; contexto domiciliar), definição dos descritores; elaboração dos critérios de inclusão e exclusão; busca na literatura e coleta de dados; analise crítica dos achados; interpretação dos achados; reportar com esclarecimentos os dados identificados.

Para nortear a referida revisão, foi estabelecido a seguinte questão "O que a literatura aborda sobre as atividades desempenhadas pelos cuidadores domiciliares no cuidado a crianças com anemia falciforme?”.

Para a construção do estudo, as coletas ocorreram nas bases de dados: Literatura Latino-Americana e do Caribe em Ciências da Saúde (LILACS); base de Dados de Enfermagem (BDENF) e portal Scientific Electronic Library Online (SciELO). Os estudos foram coletados nos períodos de junho a julho de 2021, usados os descritores "Cuidadores; criança; Anemia Falciforme", cruzados com os operadores booleanos "AND" e "OR", localizados na lista dos Descritores em Ciências da Saúde (DECs).

Foram estabelecidos os critérios de inclusão: artigos no idioma português e inglês com o recorte temporal de 2015 a 2021. E os critérios de exclusão foram: artigos duplicados, fora do recorte temporal, debates, resenhas, resumos, teses e dissertações.

\section{Resultados e Discussão}

O fluxograma representado abaixo, caracteriza a estratégia completada da busca de dados seguida pelos autores, detalhando cada etapa da pesquisa. 
Figura 1. Fluxograma com a trajetória de construção do estudo.

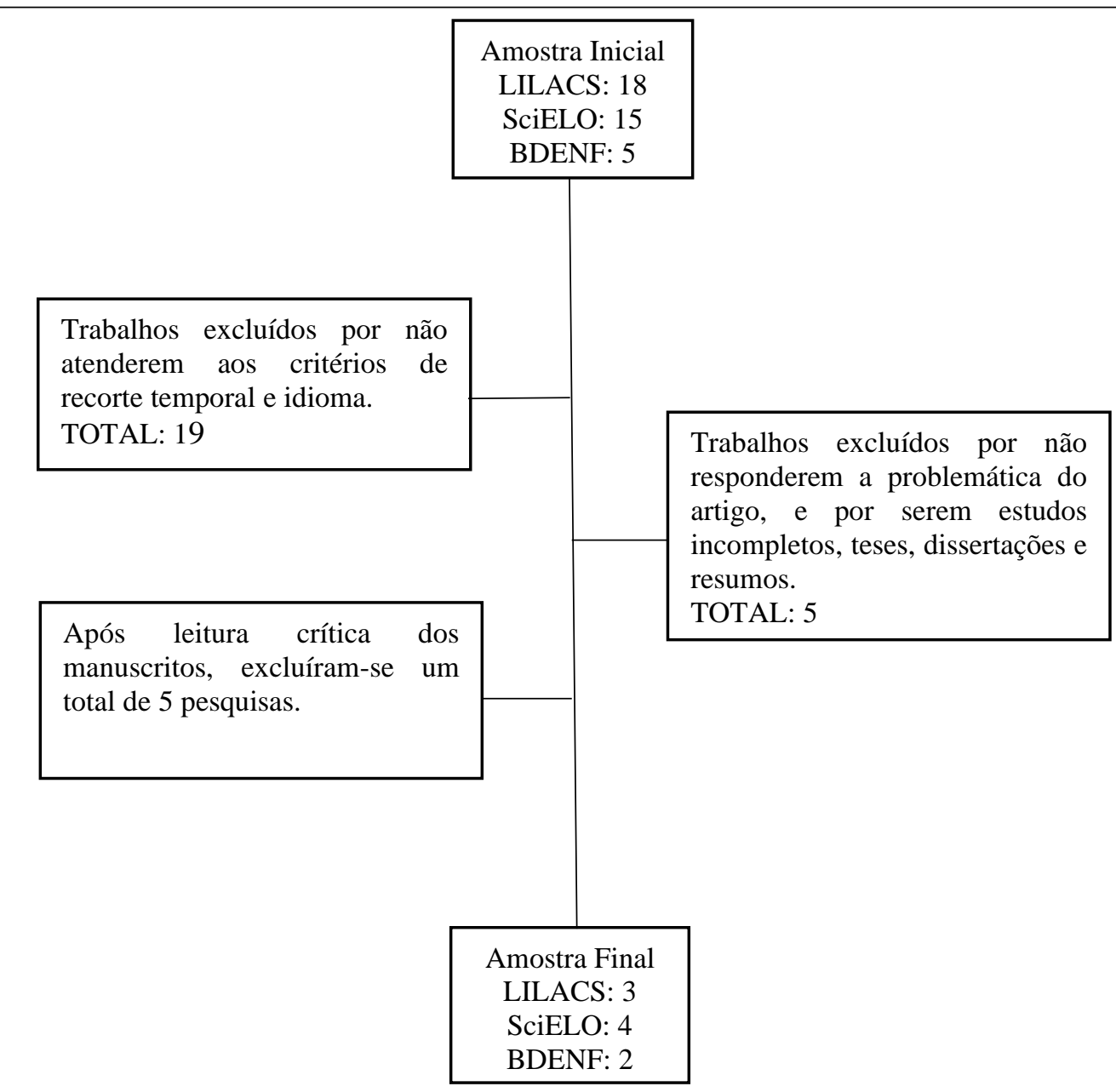

Fonte: Autores (2021).

No Fluxograma, nota-se que no início da coleta de dados, constatou-se 38 estudos, que foram submetidos a primeira etapa de avaliação por meio dos critérios de inclusão e exclusão, sendo o primeiro e segundo critério, o recorte temporal e idioma respectivamente. Assim, após aplicar os critérios de recorte temporal e idioma, foram excluídas 19 pesquisas. Os estudos foram submetidos a leitura na integra na segunda etapa, e foram excluídas 10 pesquisas por não se relacionarem com o objetivo do estudo.

Na terceira etapa, feito assim a leitura dos manuscritos, notou-se um quantitativo de 9 estudos que respondiam à questão norteadora da pesquisa.

O Quadro 1, a seguir, apresenta as pesquisas selecionadas para o estudo segundo sua autoria, título e método. 
Quadro 1 - Artigos referentes ao estudo.

\begin{tabular}{|c|c|c|}
\hline Autores & Título & Método \\
\hline Caprini \& Mota. & $\begin{array}{l}\text { O impacto psicológico em familiares cuidadores de } \\
\text { crianças e adolescentes com anemia falciforme. }\end{array}$ & $\begin{array}{l}\text { Pesquisa de abordagem quantitativa e de levantamento, } \\
\text { com amostra não probabilística de } 100 \text { cuidadores. }\end{array}$ \\
\hline Miranda et al. & $\begin{array}{l}\text { Experiência da família no manejo da criança com } \\
\text { anemia falciforme: implicações para o cuidado. }\end{array}$ & $\begin{array}{l}\text { Estudo qualitativo desenvolvido com } 14 \text { famílias de } \\
\text { crianças com anemia falciforme, mediante entrevistas } \\
\text { semiestruturadas. }\end{array}$ \\
\hline $\begin{array}{l}\text { Garioli, Paula \& } \\
\text { Enumo. }\end{array}$ & $\begin{array}{l}\text { Avaliação do coping da dor em crianças com } \\
\text { Anemia Falciforme. }\end{array}$ & $\begin{array}{l}\text { Este estudo analisou o coping da Anemia Falciforme } \\
\text { em } 12 \text { crianças com idade entre oito e dez anos, que } \\
\text { frequentavam um Hospital Universitário de Vitória, } \\
\text { Espírito Santo. }\end{array}$ \\
\hline Pacheco et al. & $\begin{array}{l}\text { O Familiar da Criança com Doença Falciforme: } \\
\text { Saberes e Práticas }\end{array}$ & $\begin{array}{l}\text { Estudo qualitativo, descritivo, desenvolvido com } \\
\text { familiares de crianças com doença falciforme, em um } \\
\text { hospital geral de Vitória por meio da entrevista } \\
\text { semiestruturada. }\end{array}$ \\
\hline $\begin{array}{l}\text { Roman, } \\
\text { Campos } \quad \& \\
\text { Bueno. }\end{array}$ & $\begin{array}{l}\text { Itinerário terapêutico como busca do cuidado ao } \\
\text { paciente com doença falciforme. }\end{array}$ & $\begin{array}{l}\text { Estudo exploratório, de abordagem qualitativa, } \\
\text { realizado por meio de entrevista semiestruturada. }\end{array}$ \\
\hline $\begin{array}{lr}\text { Fortini, } & \text { Gomes, } \\
\text { Sabóia } \quad \& \\
\text { Ferreira. }\end{array}$ & $\begin{array}{l}\text { O cuidado familiar da criança com anemia } \\
\text { falciforme. }\end{array}$ & $\begin{array}{l}\text { Estudo quantitativo, os participantes foram os } \\
\text { familiares dessas crianças. Foi utilizado um formulário } \\
\text { de entrevista estruturada. }\end{array}$ \\
\hline Figueiredo et al. & $\begin{array}{l}\text { Importância das orientações em saúde para } \\
\text { familiares de crianças com doença falciforme. }\end{array}$ & $\begin{array}{l}\text { Pesquisa qualitativa, desenvolvida em um hospital de } \\
\text { referência pediátrica no Ceara, entre abril e maio de } \\
2017 \text {. }\end{array}$ \\
\hline Rodrigues et al. & $\begin{array}{l}\text { Terapia medicamentosa no domicílio: experiências } \\
\text { de mães de crianças e adolescentes com anemia } \\
\text { falciforme. }\end{array}$ & $\begin{array}{l}\text { Estudo descritivo, com análise qualitativa dos dados, } \\
\text { realizado em setembro de } 2016 \text {. }\end{array}$ \\
\hline $\begin{array}{l}\text { Custódio, } \\
\text { Leitão, Gomes } \\
\& \text { Mendes. }\end{array}$ & $\begin{array}{l}\text { O desenhar da dor para as crianças com anemia } \\
\text { falciforme: a dor que dói, dói muito. }\end{array}$ & $\begin{array}{l}\text { Estudo exploratório, qualitativo, realizado em um } \\
\text { hospital de referência pediátrica do Ceará, com cinco } \\
\text { crianças diagnosticadas com anemia falciforme. }\end{array}$ \\
\hline
\end{tabular}

Fonte: Autores (2021).

O Quadro 2, a seguir, apresenta os estudos selecionados para a pesquisa segundo sua autoria, objetivo e ano.

Quadro 2 - Artigos tocantes ao estudo.

\begin{tabular}{|l|l|l|}
\hline Autores & Objetivo & Ano \\
\hline Caprini \& Mota. & $\begin{array}{l}\text { Investigar o impacto psicológico da anemia falciforme sobre cuidadores familiares de crianças e } \\
\text { adolescentes com a doença. }\end{array}$ & 2021 \\
\hline Miranda et al. & Compreender a experiência da família no manejo da criança com anemia falciforme. & 2020 \\
\hline $\begin{array}{l}\text { Garioli, Paula \& } \\
\text { Enumo. }\end{array}$ & $\begin{array}{l}\text { Contribuir para o estudo do coping infantil na área da Psicologia Pediátrica, este trabalho tem } \\
\text { como foco descrever e analisar o coping da dor da Anemia Falciforme em crianças com a doença. }\end{array}$ & 2019 \\
\hline Pacheco et al. & $\begin{array}{l}\text { Descrever a tipologia do cuidado realizado pelo familiar à criança com doença falciforme } \\
\text { segundo Colliére. }\end{array}$ & 2019 \\
\hline
\end{tabular}




\begin{tabular}{|l|l|l|}
\hline $\begin{array}{l}\text { Roman, Campos \& } \\
\text { Bueno. }\end{array}$ & Descrever o itinerário terapêutico de pacientes com DF na busca pelos medicamentos. & 2019 \\
\hline $\begin{array}{l}\text { Fortini, Gomes, } \\
\text { Sabóia \& Ferreira. }\end{array}$ & $\begin{array}{l}\text { Identificar o conhecimento sobre a doença falciforme na criança e descrever ações desses } \\
\text { familiares na prevenção da crise falcêmica. }\end{array}$ & 2019 \\
\hline Figueiredo et al. & $\begin{array}{l}\text { Conhecer as principais necessidades de orientação em saúde de familiares de crianças com } \\
\text { doença falciforme. }\end{array}$ & 2018 \\
\hline Rodrigues et al. & $\begin{array}{l}\text { Descrever as experiências de mães de crianças e adolescentes com anemia falciforme acerca da } \\
\text { terapia medicamentosa no domicílio. }\end{array}$ & 2018 \\
\hline $\begin{array}{l}\text { Custódio, Leitão, } \\
\text { Gomes \& Mendes. }\end{array}$ & $\begin{array}{l}\text { Compreender, por meio do desenho, as repercussões e as estratégias de enfrentamento em } \\
\text { situações ocasionadas pelas crises dolorosas da AF, a partir da perspectiva das crianças. }\end{array}$ & 2017 \\
\hline
\end{tabular}

Fonte: Autores (2021).

A anemia falciforme é a doença hereditária de maior prevalência no Brasil, acometendo cerca de 2 a $3 \%$ da população, e com quantitativos percentuais de 6 a 10\% nas pessoas negras e pardas, e reavaliando seu perfil de morbidade, observa-se altas taxas de internações hospitalares, sendo um número superior a 10 por ano, esse dado combinado a experiencias ambulatoriais com crianças de idades entre três a dez anos, contribuíram para compreensão de uma necessidade crucial de implantar estratégias de cuidado e métodos efetivos de tratamento no âmbito domiciliar (Fortini, Gomes, Sabóia \& Ferreira, 2019).

Segundo estudo de Miranda et al. (2020), o diagnóstico de AF, gera um impacto enorme a família, pois os mesmos, não possuem informações claras sobre a patologia, e em geral é descoberta com o decorrer de situações agravantes a saúde da criança, como as primeiras crises, sendo a desinformação da família um dos principais obstáculos nos primeiros meses do cuidado no domicilio, muitas vezes buscando informações em fontes não seguras, o que só comprova a também necessidade de uma educação promovida por profissionais da saúde no âmbito domiciliar a essas pessoas.

De acordo com o estudo de Figueiredo et al. (2018), a uma grande desinformação por parte dos cuidadores domiciliares de crianças com AF, contendo uma serie de afirmações errôneas, tais como: a evolução da anemia falciforme para uma leucemia, informações essas que só prejudicam o processo de cuidado, e assim destaca-se em mais um estudo, a importância de haver uma melhor interação entre profissionais de saúde e a família dos pacientes, de modo que a assistência de saúde ou especializada seja efetiva e compreendida pelos familiares.

O sentimento de amor de carinho, se torna o principal motivador para o desenvolvimento efetivo do papel de cuidador, o que contribui na busca por recursos e oportunidades para promover a criança melhores condições e qualidade de vida (Miranda et al.2020).

O cuidado no cotidiano ofertado pela assistência domiciliar se caracteriza por ser integral e amplo, abrangendo praticas em todos os eixos do indivíduo, desde ofertar uma alimentação equilibrada entre proteínas, fibras e carboidratos, á evitar ao máximo alimentos hiper lipídicos e hiper sódicos, combinando isso com uma ingesta hídrica adequada, tanto no ambiente domiciliar quanto escolar, e assim evidenciou-se também, uma preocupação com a temperatura do ambiente, agasalhando bem o filho em dias frios, e ofertando poucas roupas em dias quentes, e entre outras atividades desempenhadas cotidianamente pelo cuidador domiciliar (Pacheco et al. 2019).

No estudo realizado por Caprini \& Motta (2021), apesar da assistência continua ofertada pelo cuidador familiar, e seus medos de situações agravantes, não foi evidenciado predomínios de ansiedade ou depressão, ou seja, os familiares possuem um ajuste emocional e mental estável, o que proporciona estabilidade para desempenhar suas atividades cotidianas. 
Conforme estudos realizados por Roman, Campos \& Bueno (2019), os medicamentos para o tratamento de doença falciforme são inteiramente disponibilizados pelo Sistema Único de Saúde (SUS), e de fácil acesso, podendo ser retirado nas Unidades Básicas de Saúde (UBS) ou em farmácias distritas, porém houve grande dificuldade de seguir o tratamento medicamentoso quando os medicamentos faltavam nesses locais, e as famílias não tinham como comprar, ocasionando dependência da efetividade dessas instituições. Sendo afirmado pelo estudo de Rodrigues et al (2018), a terapia medicamentosa ofertada a crianças e adolescentes com AF, é crucial para efetividade da assistência e assim um melhor prognóstico, ocorrendo a administração diariamente também de vitamina do complexo B e folato, dentre outras medicações, para tanto, é fundamental o preparo familiar e orientação adequada para a realização correta dessas drogas no domicilio.

$\mathrm{O}$ manejo da dor em crianças com AF, é realizado de diferentes maneiras pelo cuidador, deste a administração de remédios, á atividades de distração, tais como: brincar, assistir TV, conversar e contar histórias, o que não foram efetivas quando a criança estava passando por crises álgicas (Garioli, Paula, Enumo, 2019). E assim conforme Custódio, Leitão, Gomes \& Mendes (2017), as manifestações de dor nos indivíduos com AF, devem ser tratadas com fármacos, com objetivo de regressar e melhorar o quadro clínico, aconselhando as primeiras aplicações no domicilio, se a criança não apresentar melhoras, deve-se procurar assistência hospitalar.

Como limitação a esse estudo, aponta-se as poucas pesquisas que abordam com foco as atividades e vivencias dos familiares cuidadores de crianças com doença falciforme, e assim sua perspectiva sobre essa assistência.

\section{Conclusão}

Neste estudo, ficou evidenciado, que a participação dos profissionais da saúde na educação e orientação dos cuidadores é de fundamental importância, principalmente para diminuir a quantidade de informações errôneas sobre a patologia anemia falciforme, que são facilmente disseminadas pela comunidade através de fontes não seguras de informações. Sendo imprescindível a ação dos familiares no cuidado prestado, proporcionando a criança uma ambiência adequada, alimentação saudável e administração de medicamentos quando necessário.

Ressalta-se a necessidade de uma melhor educação em saúde para essa população, pois a informação correta sobre a doença, é o primeiro "passo" para uma assistência de qualidade no ambiente domiciliar.

Essa pesquisa é importante e relevante, a maneira que estimula a produção de novos estudos nessa atemática, para que assim contribua futuramente para uma assistência mais qualificada e efetiva a esses doentes crônicos no âmbito do domicilio.

\section{Referências}

Caprini, F. R., \& motta, A. B. (2021). The psychological impact on family caregivers of children and adolescents with sickle cell anemia. Estudos de Psicologia (Campinas), 38. https://doi.org/10.1590/1982-0275202138e190168

Custódio, L. L., Leitão, I. M. T. D. A., Gomes, I. L. V., \& Mendes, L. C. B. (2017). Drawing pain for children with sickle cell anemia: the pain that hurts, really hurts. Revista Dor, 18(4). https://doi.org/10.5935/1806-0013.20170123

Dias, T. L., Oliveira, C. G. T. d., Enumo, S. R. F., \& Paula, K. M. P. d. (2013). A dor no cotidiano de cuidadores e crianças com anemia falciforme. Psicologia USP, 24(3), 391-411. https://doi.org/10.1590/s0103-65642013000300003

Eecole, Flávia Falci; Melo, Laís Samara de, \& Alcoforado, Carla Lúcia Goulart Constant. Integrative review versus systematic review. Reme: Revista Mineira de Enfermagem, v. 18, n. 1, 2014. Disponível em: https://doi.org/10.5935/1415-2762.20140001. Acesso em: 20 ago. 2021.

Favero, L., Meier, M. J., Lacerda, M. R., Mazza, V. d. A., \& Kalinowski, L. C. (2009). Aplicação da Teoria do Cuidado Transpessoal de Jean Watson: uma década de produção brasileira. Acta Paulista de Enfermagem, 22(2), 213-218. https://doi.org/10.1590/s0103-21002009000200016

Figueiredo, S. V., Lima, L. A., Silva, D. P. B. e., Oliveira, R. d. M. C., Santos, M. P. d., \& Gomes, I. L. V. (2018). Importance of health guidance for family members of children with sickle cell disease. Revista Brasileira de Enfermagem, 71(6), 2974-2982. https://doi.org/10.1590/0034-7167-2017-0806

Gravina Fortini, R., Maria Sabóia, V., De Freitas Gomes, D., \& Milton Oliveira Ferreira, A. (2019). O cuidado familiar da criança com anemia falciforme. Nursing (São Paulo), 22(250), 2734-2739. https://doi.org/10.36489/nursing.2019v22i250p2734-2739 
Research, Society and Development, v. 10, n. 11, e383101119693, 2021

(CC BY 4.0) | ISSN 2525-3409 | DOI: http://dx.doi.org/10.33448/rsd-v10i11.19693

Garioli, D. D. S., paula, K. M. P. D., \& enumo, S. R. F. (2019). Avaliação do coping da dor em crianças com Anemia Falciforme. Estudos de Psicologia (Campinas), 36. https://doi.org/10.1590/1982-0275201936e160079

Kato, G. J., Piel, F. B., Reid, C. D., Gaston, M. H., Ohene-Frempong, K., Krishnamurti, L., Smith, W. R., Panepinto, J. A., Weatherall, D. J., Costa, F. F., \& Vichinsky, E. P. (2018). Sickle cell disease. Nature Reviews Disease Primers, 4(1). https://doi.org/10.1038/nrdp.2018.10

Miranda, F. R. D., Ivo, M. L., Teston, E. F., Lino, I. G. T., Mandetta, M. A., \& Marcheti, M. A. (2020). Experiência da família no manejo da criança com anemia falciforme: implicações para o cuidado. Revista Enfermagem UERJ, 28, Artigo e51594. https://doi.org/10.12957/reuerj.2020.51594

Ministério da Saúde reforça a importância da deteç̧ão da Doença Falciforme. (2020, 19 de junho). Ministério da Saúde. https://www.gov.br/saude/ptbr/assuntos/noticias/ministerio-da-saude-reforca-a-importancia-da-deteccao-da-doenca-falciforme

Pacheco, D. P., Da Costa, B. C., Nunes Nascimento, L. D. C., De Souza, T. V., Bastos Depianti, J. R., \& Laignier, M. R. (2019). Relatives of Children Bearing Sickle Cell Anemia: Knowledge and Practice / O Familiar da Criança com Doença Falciforme: Saberes e Práticas. Revista de Pesquisa: Cuidado é Fundamental Online, 11(5), 1213. https://doi.org/10.9789/2175-5361.2019.v11i5.1213-1218

Roman, C., Campos, M. d. S., \& Bueno, D. (2020). itinerário terapêutico como busca do cuidado ao paciente com doença falciforme. Revista Baiana de Saúde Pública, 43(3), Artigo 10.22278/2318-26

Silva-Rodrigues, Fernanda Machado, Oliveira, Natasha S. Sales de, Leite, Ana Carolina Andrade Biaggi, Nunes, Michelle Darezzo Rodrigues, Polita, Naiara Barros, \& Nascimento, Lucila Castanheira. (2018). terapia medicamentosa no domicílio: experiências de mães de crianças e adolescentes com anemia falciforme. Cogitare Enfermagem, 23(2), e53462. Epub 21 de janeiro de 2019.https://dx.doi.org/10.5380/ce.v23i2.5346260.2019.v43.n3.a2762.

UOL. (2019, 23 de abril). Apesar de tratamento avançar, doenças falciformes crescem em países pobres. UOL - Seu universo online. https://www.uol.com.br/vivabem/noticias/redacao/2019/04/23/apesar-de-tratamento-avancar-doencas-falciformes-crescem-em-paisespobres.htm?next=0001H1836U11N 